\title{
Undergraduate Research Projects using Microfluidic Devices
}

\section{Dr. Irina Nicoleta Ciobanescu Husanu, Drexel University (Tech.)}

Dr. Ciobanescu Husanu is Assistant Professor in Mechanical Engineering Technology at Drexel University. She received her PhD degree in mechanical engineering from Drexel University and also a MS degree in aeronautical engineering. Her research interest is in thermo-fluid sciences with applications in micro-combustion, fuel cells, green fuels and plasma assisted combustion. Dr. Husanu has prior industrial experience in aerospace engineering that encompasses both theoretical analysis and experimental investigations such as designing and testing of propulsion systems including design and development of pilot testing facility, mechanical instrumentation, and industrial applications of aircraft engines. Also, in the past 8 years she gained experience in teaching ME and ET courses in both quality control and quality assurance areas as well as in thermal-fluid, energy conversion and mechanical areas from various levels of instruction and addressed to a broad spectrum of students, from freshmen to seniors, from high school graduates to adult learners. She also has extended experience in curriculum development.

\section{Dr. Radian G Belu, Drexel University (Tech.)}

Dr. Radian Belu is Assistant Professor within the Engineering Technology (ET) program - Drexel University, Philadelphia, USA. He is holding the second position as Research Assistant Professor at Desert Research Institute - Renewable Energy Center, Reno, Nevada. Before joining to the Drexel University Dr. Belu hold faculty and research positions at universities and research institutes in Romania, Canada and United States. He also worked for several years in industry as a project manager and senior consultant. He has taught and developed undergraduate and graduate courses in electronics, power systems, control and power electronics, electric machines, instrumentation, radar and remote sensing, numerical methods and data analysis, space and atmosphere physics, and physics. His research interests included power system stability, control and protection, renewable energy system analysis, assessment and design, power electronics and electric machines for wind energy conversion, radar and remote sensing, wave and turbulence simulation, measurement and modeling, numerical modeling, electromagnetic compatibility and engineering education. During his career Dr. Belu published several papers in referred journals and in conference proceedings in his areas of the research interests. He has also been PI or co-PI for various research projects United States and abroad in power systems analysis and protection, load and energy demand forecasting and analysis, renewable energy analysis, assessment and design, turbulence and wave propagation, radar and remote sensing, instrumentation, atmosphere physics, electromagnetic compatibility, and engineering education.

Mr. Patrick Lee Kirby, Drexel University (Eng.)

Mr. Justin Bryan Gillander 


\title{
Undergraduate Research Projects using Micro-fluidic Devices
}

\begin{abstract}
In order for students to enhance their understanding of engineering concepts, hands-on experience proves essential. Especially when the topic of interest involves heat transfer, fluid dynamics or both, it becomes very difficult for students to obtain a hands-on experience due to the nature of and the costs associated with the experimental apparatus. Incorporating the design component in undergraduate engineering education has been an immediate and pressing concern for educators, professional societies, industrial employers and agencies concerned with national productivity and competitiveness. Student-led projects as required components of course curriculum add tremendous value to science and engineering education. The design experience through project work develops the students' lifelong learning skills, self-evaluations, selfdiscovery, and peer instruction in the design's creation, critique, and justification, providing them with several learning opportunities that cannot be taught in a class or laboratory. Cost and space issues can be addressed by the current trend in engineering for miniaturization and microscale systems, systems that can be readily designed and prototyped by students.

This paper discusses the development and implementation and educational outcome assessment of two student projects using micro fluidic devices, involving a number of junior and senior undergraduate students at our engineering technology program. The goal of the design projects is to explore and enhance students understanding of the fundamental engineering principles, and hands-on demonstration of system prototyping. Here we report two micro-fluidic projects which explore the principles of heat transfer as well as mixing phenomena with potentially obtaining a turbulent flow in a micro fluidic device.

The first project incorporates a micro-heat exchanger, mimicking a plate and tube heat exchanger. Students explored the heat transfer phenomena and the temperature dependence over time. The data were acquired and monitored using temperature and pressure digital sensors and a LabVIEW VI interface. This project was used as experimental set-up for further enhancement of laboratory activities for ET courses related to heat transfer, measurement and instrumentation and fluid mechanics. The second project one is a piezo-micro-fluidic micro-mixing device, with the primary mixer being a pump, which will generate adequate mixing in micro fluidic channels.
\end{abstract}

\section{Introduction}

Engineering Technology programs, which in contrast to traditional engineering programs, place greater emphasis on practical applications, hands-on learning, and engineering theory related to energy and energy conversion, manufacturability, maintainability, reliability, and quality assurance. Engineering Technology graduates often specialize in prototyping, instrumentation, statistical process control, and manufacturing operations. They develop the skills needed for deployment and implementation of sophisticated devices and systems in the field, and closely interact with end-users of technology.

In order for students to enhance their understanding of engineering concepts, hands-on experience is essential. Especially when the topic of interest involves heat transfer, fluid dynamics or both, it becomes very difficult for students to obtain a hands-on experience due to 
the nature of the experimental apparatus. Incorporating the design component in undergraduate engineering education has been an immediate and pressing concern for educators, professional societies, industrial employers and agencies concerned with national productivity and competitiveness. Student-led projects as required components of course curriculum add tremendous value to science and engineering education. The design experience develops the students' lifelong learning skills, self-evaluations, self-discovery, and peer instruction in the design's creation, critique, and justification. The experience, which would be difficult to complete individually, gives students the accomplishment that is often lacking in many engineering courses, using traditional teaching approaches, motivating student learning and developing the industrial required skills required. Only project work gives them the opportunity to become a problem solver or an innovator. Hence, project work is a very important constituent of our engineering technology curriculum. Project work provides several opportunities for students to learn several aspects of importance to an engineer that cannot be taught in a class or laboratory. However, designing and prototyping real industrial sub-systems or components can become very costly, and also may require additional laboratory space. Some of these issues can be addressed by the current trend in engineering for miniaturization and micro-scale systems. These systems can be readily designed and prototyped by students.

The Engineering Technology Program at our university in particular represents an attractive option for technically-inclined students whose interests and motivations center on practical applications, manufacturing, hands-on problem solving, and intuitive and visual thinking; and for whom the abstract theory and analysis emphasized in traditional engineering courses of study proves to be a disincentive. This type of student is a valuable asset to industry and such students may forego a career path in science and engineering unless options such as Engineering Technology are open to them. Due to its straightforward conceptual basis, amenability to visual demonstrations, accessibility of the prototyping technology to students, and its impressive applications in the biomedical area, micro-fluidics offers an excellent means for attracting students to science and engineering ${ }^{1-7}$.

Micro-fluidics has become an important commercial technology and engineering discipline in its own right, with extensive applications in biotechnology, medicine, chemistry, materials science, nanotechnology, and energy conversion. Micro-fluidics also provides an outstanding vehicle to educate and expose students to engineering subjects that are hallmarks of Engineering Technology curricula. Further, the instructional materials and resources developed here will have widespread applicability and broad interest to engineering students in general, and will also serve the needs of students in engineering technology, applied sciences, biotechnology, biomedical engineering and healthcare technology programs. Micro-fluidics can be an effective 'gateway' to biotechnology and biomedical fields for students with otherwise little background in biology. Also, new applications of micro-fluidics have been developed related to energy storage and energy conversion fields, mostly in renewable energy areas ${ }^{6}$. Although micro-fluidic devices for energy conversion are still in the research and development stage, their basic operating principles may form excellent educational tools in exploring and understanding physical concepts related to thermo-fluids and heat transfer fields.

The main objective of this paper, stemmed from the two-year awarded NSF TUES project, is to present some of the results of integration of micro-fluidics technology and applications into Engineering Technology (ET) curriculum. We report about the incorporation of micro-fluidics 
related student-led projects in two courses: "Thermodynamics and Heat Transfer Laboratory" course and "Fluid Power" course. These courses were offered in the winter and spring terms of AY 2011-2012, and in fall and winter terms of AY 2012-2013. The immediate objective has been achieved by a combination of experimental activities and demonstrations, with emphasis on aspects of micro-fluidic technology related to micro-scale fluidics, heat transfer, and instrumentation and control, with a brief introduction to design of experiments (DOE) ${ }^{12-16}$.

During the past and current AY, couple laboratory activities related to micro-fluidics were developed and implemented and as a result of these activities, students being exposed to various aspects of micro-fluidics devices and technologies. Based on student responses at the end of winter and spring terms of AY 2011-2012 we concluded that students believed that the projects provided an excellent opportunity to expand their understanding of the topics regarding micro-fluidic devices, while applying the fundamental principles of thermodynamics, heat transfer and fluid mechanics. One of the main assessment tools for both courses was a student team project based on either an instructor suggested topic or a topic of their choice. Two teams (one from each course) chose their projects to be in the microfluidic area and also chose to expand their project work beyond the course requirements, developing the prototype of a further lab activity for further courses with same topic. Students were assessed continuously during the development of the projects. The assessment focused on various aspects regarding their level of understanding of the fundamental principles, and the level of applying science to an engineering technology problem. These projects demonstrated the students' mastery of the techniques and knowledge learned during the course. Students were able to apply the current engineering technology procedures and methods to a new and challenging engineering system, producing a practical solution based on detailed analysis of the system requirements.

\section{Heat Exchanger project}

First project is related to Thermo and Heat transfer course topics and was aimed at developing a micro-fluidic heat exchanger to be further used as a laboratory experimental set-up and lab activity in the future.

Thermodynamics and Heat Transfer course is a three hour-credit junior to senior undergraduate core curriculum course designed for Mechanical Engineering Technology (MET) students. Also, this course is one of the main precursors of Senior Design capstone course, having embedded in its curricula, besides seven laboratory activities, a final student-led team project that has the main outcome, a working prototype of a thermo-fluid system. The unique character of this course consists in being the only exposure that our students will have to heat and mass transfer phenomena through laboratory activities enhanced by lectures. Laboratory activities account for two thirds of the course, while the classroom-lecture part, only for one third. The material conveyed primarily, is using hands-on approaches.

During this course students will explore experimentally basic thermodynamic relations; they will conduct experiments related to flow of compressible fluids and to energy conversion of a fuel into a working substance and the related heat transfer mechanisms ${ }^{13,}{ }^{14}$. The main course objectives (lectures and labs combined) are focused on using heat transfer principles to understand the behavior of thermal-fluid systems, on illustrating the development of the governing equations associated with thermal systems, on investigating the influences of various 
system parameters and conditions on the resulting steady or transient response of the system, on providing students with the basic tools used in thermal system design, and on exposing students to heat transfer applications in industry. The topics covered in this course range from a review of basic thermodynamic concepts and principles (First and Second Law of thermodynamics, Energy Balance, Entropy), to heat and mass transfer concepts (basic modes of heat transfer, heat conduction, convection (natural and forced), and radiation), with applications for heat exchangers.

This project, developed by two students in the "Thermo and Heat Transfer" course, was designed to incorporate a micro-fluidic heat exchanger. The heat exchanger is mimicking a plate and tube heat exchanger configuration, using two micro-pumps for driving the two flows (cold and hot). Students explored the heat transfer phenomena and the temperature dependence over time. The data acquisition and monitoring system used temperature and pressure digital sensors controlled by a LabVIEW VI interface. This project was developed as a class project and further improved beyond class to be used as experimental set-up of laboratory activities. The activities envisioned were for courses related to heat transfer, measurement and instrumentation, and fluid mechanics.

The characteristics of the system presented below are studied and developed from concept to final prototype by the students under faculty supervision. As can be easily observed, students described and in-depth analyzed various phenomena associated with creating a heat exchanger and also with those associated with a micro-fluidic device. Students used initially a 3-D printer to create the first prototype. The second system was milled from an acrylic transparent plate for a better visualization of the flow. Also, students used an improved instrumentation design for the second system, allowing a better monitoring of the temperature variation over the fluid flow in both streams.

The type of heat exchanger that was created for this project is based on the idea of combining the concepts of a plate heat exchanger with a plate and tube micro-fluidic heat exchanger. This heat exchanger uses different metal plates in between the two fluids to create the transfer of heat. The plated offer a major advantage of creating a large heat transfer surface area (see Figure 1and 2).

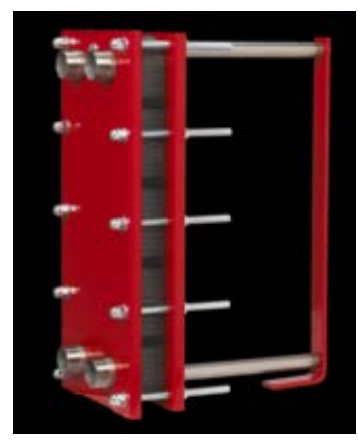

Figure 1: Interchangeable plate heat exchanger

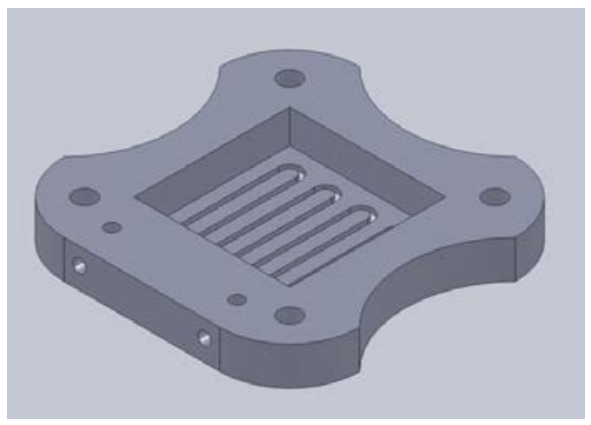

Figure 2: Heat Exchanger Design

Students used SOLIDWORKS to create their concept design and 3D printing technology and a 3D printer to create their first prototype underlining the interdisciplinary character of this endeavor (Figure 2). Conceptually, they used their heat exchanger design concepts learned during the course, such as theoretical calculations of temperature profiles for different configurations (parallel or counterflow), particularized for the type of heat exchanger chosen, 
estimates of Reynolds and Nusselt numbers, and also the variation of these parameter as a function of relative position of the upper plate versus the lower plate. All these considerations were evaluated in connection with the restrictions associated with a micro-fluidic device, such as a low Reynolds number and bubble formation along the tubes, as well as the difficulties connected with fabrication, instrumentation and sealing of a very small device. As it can be seen from Figure 2, each plate has the tubing in the middle and a cut out, with a window for the easiness of observation of the two fluids moving through the system. The two plates will be held together by four bolts place in the four holes created in each corner. Smaller holes were created on the top for placing thermocouples that will monitor the input and output temperature of both fluids. The holes on the side are for the inflow and outflow tubing.

Figure 3 depicts the inner plates that will facilitate the heat transfer between the two flows (hot and cold) and also they will separate the two flows. In Figure 4 we present the overall schematic of the experimental set-up used for this heat exchanger. The plate used for the interface between the two flows is made of 0.005 " Copper and that can be changed for either 0.005" Brass or 0.005 ” PVC. These different plates were used to study the influence of the material properties upon heat transfer between hot and cold flow. Data acquisition was realized using a LabVIEW VI and a NI USB DAQ 6009 interface (Figure 5).

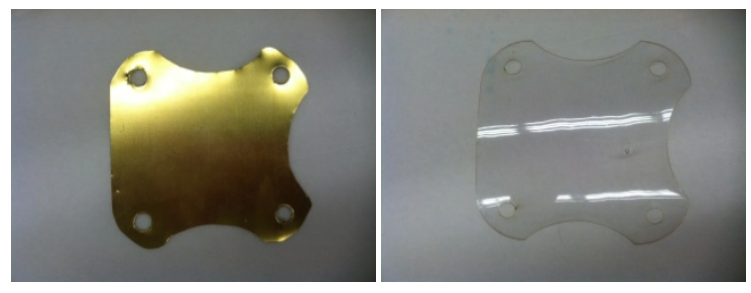

Figure 3: Brass and PVC Material

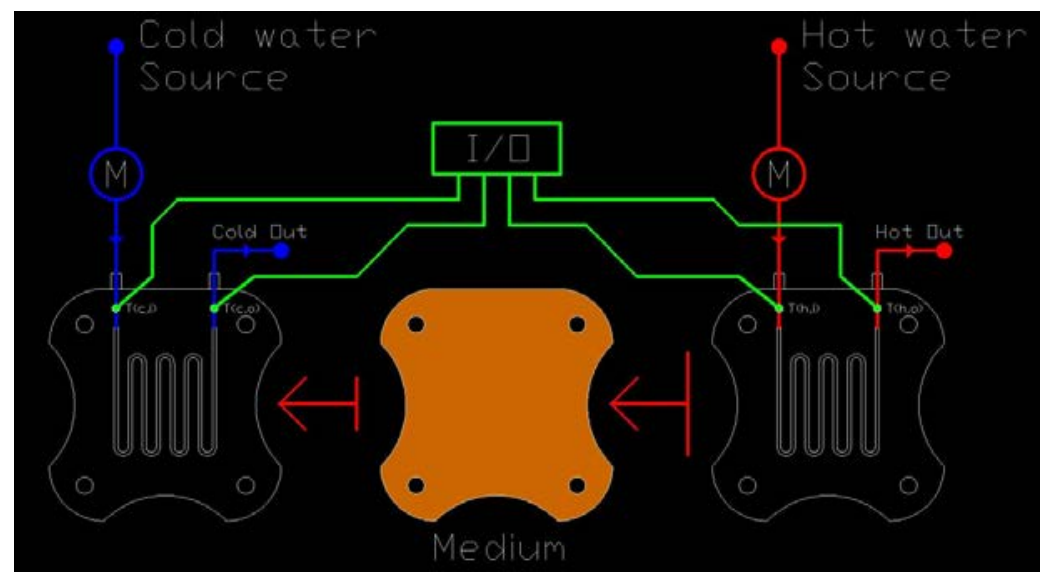

Figure 4: Set up of the Experiment 


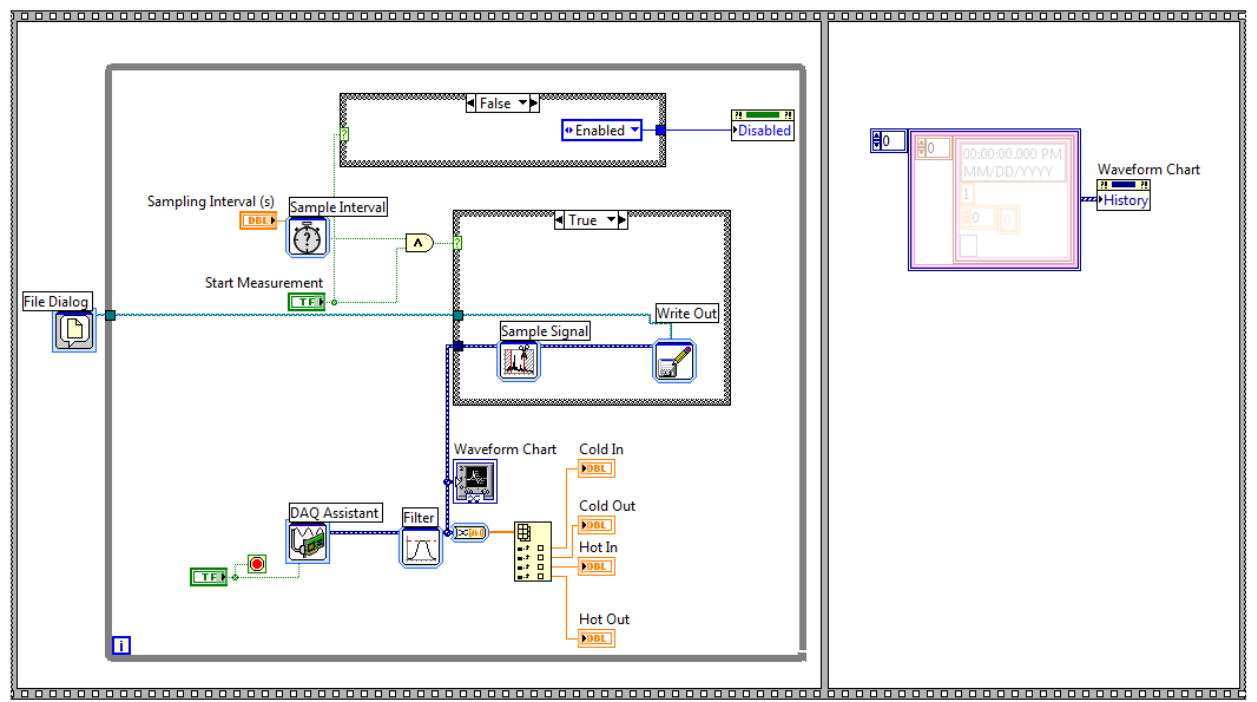

Figure 5: LabVIEW VI

The fully assembled microfluidic heat exchanger set-up is presented in the Figure 6 below.

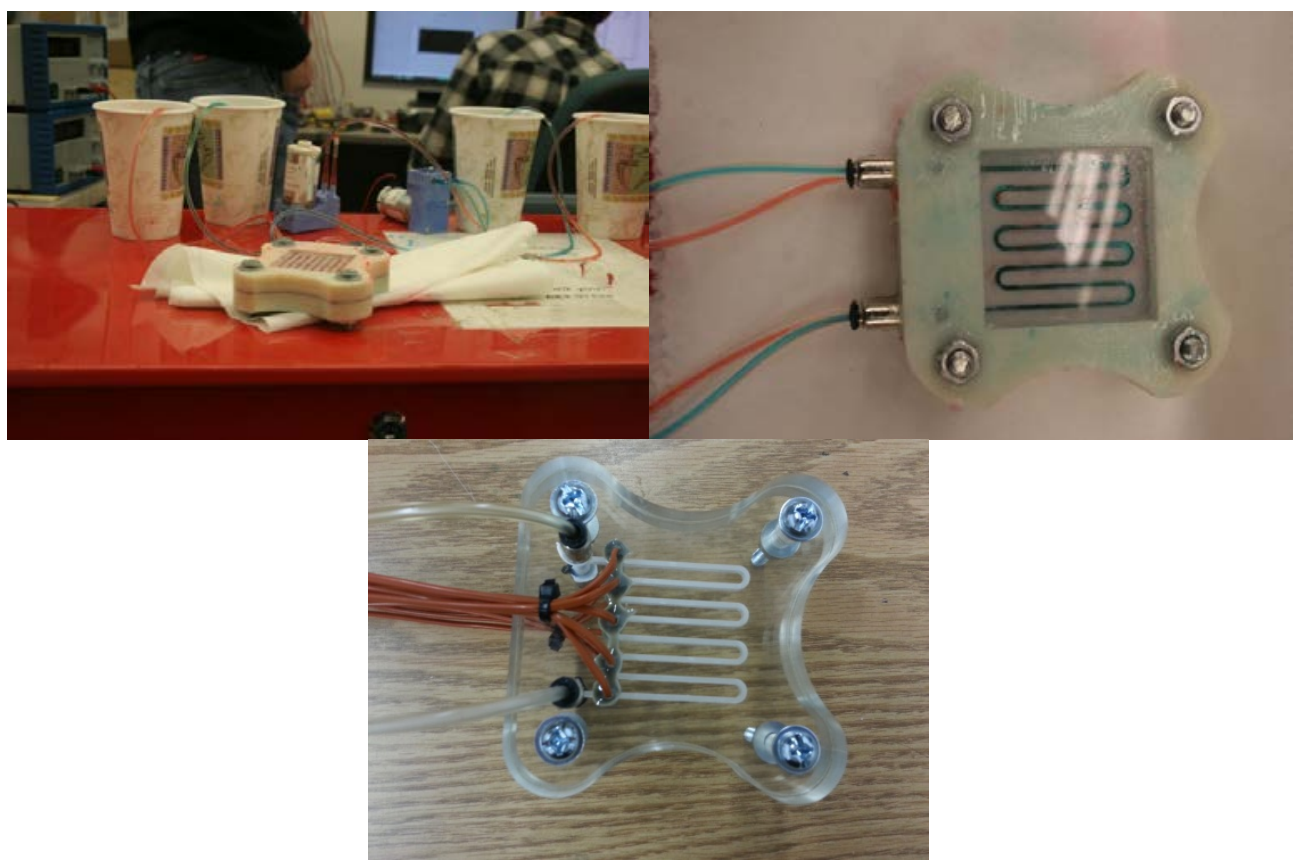

Figure 6 Micro-fluidic heat exchanger assemblies: top left and right - initial design, bottom - improved design

The following tabulated and graphed data are obtained using calculated temperature averages using the equations (1) through (5) presented below:

$$
T_{c, \bar{\zeta} \boldsymbol{\alpha}, \mathrm{vg}}=\frac{\sum T_{c, i}}{n}
$$

$$
T_{c, o, a v g}=\frac{\sum T_{c, o}}{n}
$$




$$
T_{h, j \mathrm{avg}}=\frac{\sum T_{h, i}}{n}
$$

$$
T_{h, o, \text { vgg }}=\frac{\sum T_{h, o}}{n}
$$

Where $T_{c, i}$ and $T_{c, o}$ are the temperature at inlet and outlet for cold flow and $T_{h, i}$ and $T_{h, o}$ are the temperature at inlet and outlet for hot flow, and $\mathrm{n}$ is the number of samples taken.

The effectiveness values ( $(\boldsymbol{\varepsilon})$ are calculated using eqn. (5) for both parallel flow and counter flow because in none of the tests $\mathbf{T}_{\mathbf{c}, \mathbf{0}}$ value was greater than $\mathbf{T}_{\mathbf{h}, \mathbf{0}}$.

$$
\varepsilon=\frac{T_{h, i}-T_{h, o}}{T_{h, i}-T_{c, o}}
$$

With the calculated temperature averages you can then proceed to calculate the effectiveness of each of the tested systems.

Students created the system flexible enough to be amenable for various configurations and to enable them to study the influence of each configuration on temperature profile, Nusselt number, local convection coefficient, and effectiveness. Figures 7, 8 and 9 are a sample of students' analysis and validation of their innovative design. These figures illustrate the temperature profiles for different plate interfaces and also different flow configurations, along the length of the tube. On abscissa (x-axis) is the length of the tube between inlet (1) and outlet (2), and on ordinate (y-axis) is the temperature. In the data tables presented above the graphs students summarized observed data acquired via instrumentation and their respective calculated heat exchanger parameters such as temperature differences and effectiveness.

Overall, these figures below (Figures 7, 8, and 9) demonstrates the flexibility and the versatility of the system as well as the students' practical understanding of the complexity involved in designing a laboratory set-up for studying a heat exchanger.

\begin{tabular}{|r|r|}
\hline Copper - Parallel Flow & \\
\hline$T(c$, in $)=4.99$ & $\Delta T($ cold $)=16.15$ \\
\hline$T(c$, out $)=21.14$ & $\Delta T($ hot $)=20.91$ \\
\hline$T(h$, in $)=61.14$ & $\mathrm{Cr}=0.77$ \\
\hline$T(h$, out $)=40.23$ & $\varepsilon=0.37$ \\
\hline
\end{tabular}

\begin{tabular}{|r|r|}
\hline Copper - Counter Flow & \\
\hline$T(\mathrm{c}$, out $)=23.23$ & $\Delta \mathrm{T}($ cold $)=16.92$ \\
\hline$T(\mathrm{c}$, in $)=6.31$ & $\Delta \mathrm{T}(\mathrm{hot})=26.70$ \\
\hline $\mathrm{T}(\mathrm{h}$. in $)=68.17$ & $\mathrm{Cr}=0.63$ \\
\hline $\mathrm{T}(\mathrm{h}$, out $)=41.47$ & $\varepsilon=0.43$ \\
\hline
\end{tabular}
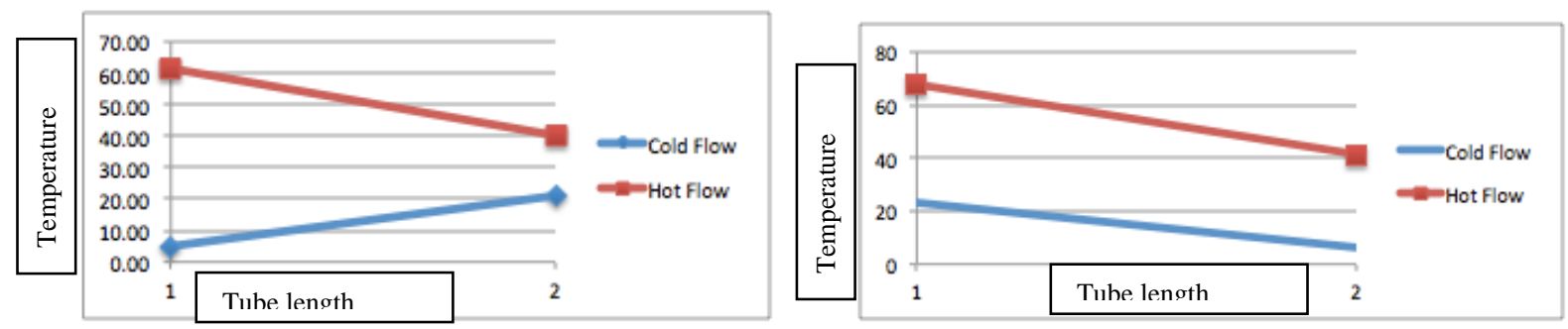

Figure 7: Temperature graph and data for copper in parallel flow (right) and counter flow (left) 
Brass - Parallel Flow

$T(\mathrm{c}$, in $)=5.51 \quad \Delta \mathrm{T}$ (cold) $=16.92$

$T(c$, out $)=22.43 \quad \Delta T($ hot $)=25.26$

$\mathrm{T}(\mathrm{h} . \mathrm{in})=67.52 \quad \mathrm{Cr}=0.67$

$T(h$, out $)=42.26 \quad E=0.41$

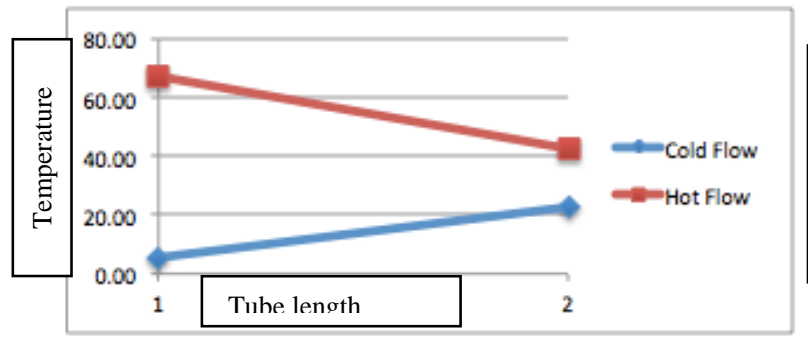

Brass - Counter Flow

$\mathrm{T}(\mathrm{c}$, out $)=19.92 \quad \Delta \mathrm{T}($ cold $)=17.11$

$T(\mathrm{c}$, in $)=2.81 \quad \Delta \mathrm{T}($ hot $)=20.48$

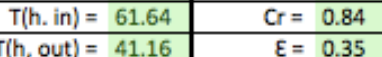

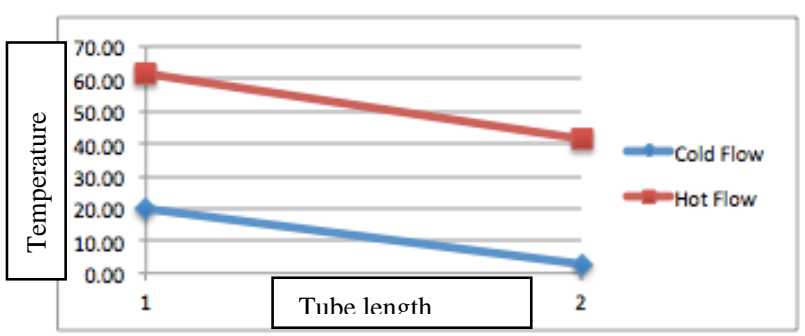

Figure 8: Brass - Parallel and counter flow temperature charts

\begin{tabular}{|r|r|}
\hline PVC - Parallel Flow & \\
\hline$T(\mathrm{c}$, in $)=2.59$ & $\Delta \mathrm{T}($ cold $)=10.56$ \\
\hline$T(\mathrm{c}$, out $)=13.15$ & $\Delta \mathrm{T}($ hot $)=13.09$ \\
\hline$T($ h. in $)=64.58$ & $\mathrm{Cr}=0.81$ \\
\hline$T($ h, out $)=51.49$ & $\varepsilon=0.21$ \\
\hline
\end{tabular}

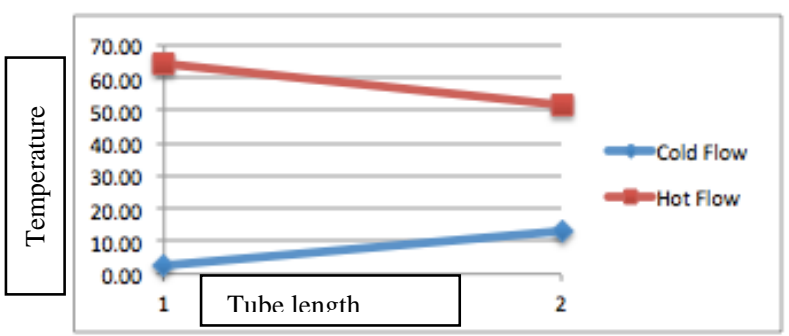

\begin{tabular}{|c|c|}
\hline PVC - Counter Flow & \\
\hline$T(c$, out $)=10.77$ & $\Delta \mathrm{T}($ cold $)=7.07$ \\
\hline$T(c$, in $)=3.70$ & $\Delta T($ hot $)=11.41$ \\
\hline$T(h$. in $)=60.77$ & $\mathrm{Cr}=0.62$ \\
\hline$T(h$, out $)=49.36$ & $\varepsilon=0.20$ \\
\hline
\end{tabular}

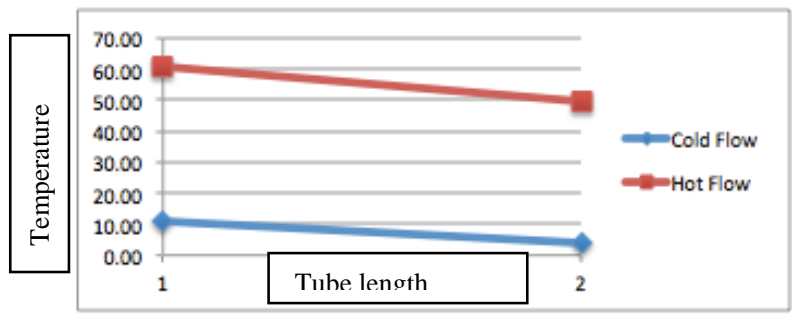

Figure 9: PVC- Parallel and counter flow temperature charts

After testing and data analysis, students concluded that in general, counter flow will have a higher effectiveness than parallel flow. We can see this trend with copper, as the effectiveness with parallel flow is 0.37 and 0.43 with counter flow. Unfortunately, both the Brass and PVC mediums did not follow this trend. This led students to conclude that they have to further improve the accuracy of their heat exchanger.

Students performed a detailed analysis of the correlation between effectiveness and the flow configuration relative to the plate used. Therefore they concluded that, with the same device, copper is a superior material to use in heat exchangers and that the PVC being an insulator will determine low effectiveness of this type of heat exchanger. During the development, testing and data analysis of this system, students were exposed to a variety of concepts ranging from basic fluid mechanics, heat and mass transfer and manufacturing, all of them related to microfabrication and functionality of a micro-fluidic device. They also presented in the term project the hurdles that they encountered in developing it and also proposed some improvements that will produce a better accuracy of the results.

Students decided to further develop this project outside the class time and the course framework and to create a valuable experimental set-up to be used in various other courses including in "Thermo and Heat Transfer" course. This newly developed heat exchanger has been used during winter term 2013 for the same course. For this purpose, they built a new and 
improved micro-fluidic heat exchanger using a better quality material (such as nylon), and better manufacturing process (plates were machined on a CNC mill instead of 3D printed).

They improved thermocouples' mountings and also they added more thermocouples along each flow to better characterize the flow temperature profile. For the new device they are using actual water flow motors with low flow rates and in-flow flow-meters.

Some of the hurdles described by the students in their project are related to the inaccuracies of the device (it is known that with the same device, counter flow should have a higher effectiveness that parallel flow). However, this can be due to many design and manufacturing problems that they could not solve in the allotted time. They developed an improved model, trying to eliminate most of the above mentioned issues. Some problems were related to the porosity of the material used, resulting in many leaks in the device while running the test. Another problem rose from the insertion points of the thermocouples as they may shift or separate from the main plate, especially when changing the mediums. To solve these two problems they manufactured the new plates using a 5-axis CNC machine manufacture the heat exchanger out of solid block of nylon. The nylon is not porous and is more elastic than the 3D printer material, so is more resistant to cracks and fissures. Also nylon is a better insulator than the 3D printing material. Machining the part provides considerably more accurate tolerances, giving better estimates for the area and flow rate of the device. Also, the thermocouples are permanently mounted into the device using epoxy adhesive, preventing the tips from moving or sliding out. The micro-pumps are able to run a very low flow rates, increasing the effectiveness of the heat exchange process itself, as the previously used pumps did not handle well low flow rates. Another improvement was to mount in the system "in-flow" flow-meters, for a better accuracy of estimating the performances of the device.

Therefore, based on students' analysis and inferences related to process testing and validation, and improvement, we may conclude that students demonstrated their ability to design, manufacture and test a micro-fluidic heat exchanger, and also to integrate in this project knowledge acquired in various disciplines (interdisciplinary character of the project). They proved that they understood in-depth the topics presented in the course and reinforced through laboratory experiments. It can be easily inferred from the above analysis that students creatively developed a miniaturized laboratory set-up amenable for further investigations by other students, an experimental setting that is used currently to illustrate the basic principles of heat transfer and heat exchanger design. What is commendable is the ability of the students to detect the problems that arose from developing this system and being able to find solutions to these problems. This learn by doing process is extremely valuable for students' academic progress and for their preparation for an engineering career. They combined successfully both aspects comprised in this project: to develop a micro-fluidic heat exchanger and an educational laboratory set-up that will serve as learning material for other students. They correctly identified the important aspects of the course principles, concepts and notions that might and needed to be illustrated by this system, and they came with a practical solution: the presented system.

\section{Piezo-micro-fluidic micro-mixing device}

The second project is a piezo-microfluidic micro-mixing device, with the primary mixer being a pump, which will generate adequate mixing in microfluidic channels. The piezoelectric effect is

useful in allowing generation of high frequency resonance, which results in the contraction and 
expansion of the piezoelectric material, causing a bending stress in the ceramic material. The bending stress causes turbulence and promotes mixing within the designed chip. This project was developed as part of the Fluid Power course, during the winter term 2012. Also this project was continued outside course framework for improvement and further development to be included as laboratory activity and experimental set-up for thermal-fluid laboratory classes.

The Fluid Power curse covers the fundamentals of hydraulic/pneumatic systems with an emphasis on applications of Bernoulli's equation. Topics include component types and designs, hydraulic/pneumatic circuit analysis and design of hydraulic/pneumatic systems. Fluid power systems can be classified as hydraulic systems (liquid is the power transmitter) and pneumatic systems (air is the working fluid).

As with various other areas of science and technology, smaller seems to be "the way to go" with practically every new advance in many different fields. One can assume then, that microfluidics (fluids that operate in the micro-scale environment) play just as an important role as traditional fluids on the macro scale. By being able to manipulate and control fluids on the micro-scale, creates the potential for groundbreaking developments in biotechnology, chemistry, and various other engineering fields, having the ability to become a vital player in the numerous industrial applications. Some of the most commercially successful applications of microfluidics include the inkjet print head. The inkjet print-head also incorporates the use of a piezoelectric actuator (Inkjet Printer). Students examined in-depth the principles of piezoelectric actuator throughout this project.

The main objective of this student project was to study the micro-mixing methods and possibilities under the constraints of micro-devices. Micro-mixing is a key component in many different technical, analytical and manufacturing applications. Rapid micro-mixing can also reduce the amount of sample needed as well as reduce the time and the cost of sample analysis. For these reasons, micro-mixing is an extremely important field of microfluidics that needs to be examined. Although currently several investigations are examining micro-mixing phenomena, no leading technology have been developed in this field may be used with most applications. Currently, the major obstacle in microfluidic research is the problem of successfully creating mixing in the fluids themselves ${ }^{17-20}$. Several potential solutions have been investigated and reported in the literature regarding micro-mixing, such as magnetic mixing, electrokinetically driven mixing, chaotic mixing etc. Generating turbulence within a micro-fluidic device is not practical for micro-mixing since microfluidic devices generally promote laminar flow as opposed to turbulent flow, due to very small flow channels (micro size or less) ${ }^{24}$. There are many different areas that can be investigated to promote micro-mixing. As part of their project students carried out an extensive literature search in this field, and they explored and tested magnetic micro-mixing using a magnetic fluid and a stirrer. Students chose to create mixing using a newly developed micro-mixing device with a micro-pump as a primary mixer, which will generate adequate mixing in microfluidic channels.

Due to time constraints, this mechanical apparatus was not yet physically prototyped, though the mathematical probabilities and potential improvements, as well as a conceptual design, have been discussed by students in their project, as presented below.

The first step in designing this experimental apparatus is to determine what type of micro-pumps they need for this microfluidic device. There are over "200 archival journal papers reporting new 
micro-pumps or analyzing micro-pump operation”20. Most operational micro-pumps are reciprocating displacement micro-pumps where a "moving boundary/surface does pressure work on the given fluid in a systematic, timely manner" ${ }^{20}$. During the literature survey regarding micro-pumps students found that for pump construction it is used mostly silicon, glass or a combination of both, and that most pumps have a piezo-electric driver. Most also use a type of flap system for the inlet and outlets of the pump.

They chose to investigate a design created by Gonzalez et al. (Figure 10) for the design of the micro-mixing device. In the original design a piezoelectric (lead zirconium titanate or PZT) is attached to the membrane (silicon nitride), which causes turbulence within the channels of the device. The piezoelectric effect is useful in allowing generation of high frequency resonance, which results in the contraction and expansion of the piezoelectric material, in this case PZT, causing a bending stress in the $\mathrm{Si}_{3} \mathrm{~N}_{4}$ ceramic material. The bending stress will cause turbulence and promote mixing in the chip. For these reasons, piezoelectric materials are considered for the conceptual design.

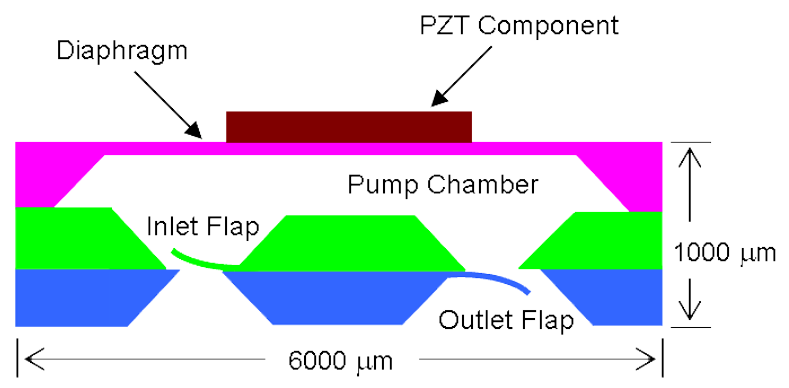

Figure 10: Schematic for micro-pump using piezoelectric component

The materials chosen for the prototype are PZT5A and $\mathrm{Si}_{3} \mathrm{~N}_{4}$. The PZT5A was chosen because, similar to other PZT components, it has the ability to generate substantial piezoelectricity when minimal deformation occurs. The $\mathrm{Si}_{3} \mathrm{~N}_{4}$ ceramic was selected because of its high strength, elastic modulus and fracture toughness. The constraints for the design are limited by several internal and external factors; amongst yield strength is one of most important factor. To achieve the desired bending of the material, a relatively high elastic modulus as well as high yield strength is desired for the boundary layers. $\mathrm{Si}_{3} \mathrm{~N}_{4}$ is an extremely common ceramic used in microfluidic applications because of its high yield strength and high elastic modulus. Another experimental design was considered as well and is based on the design created by Moussa et al. (Figure 11).

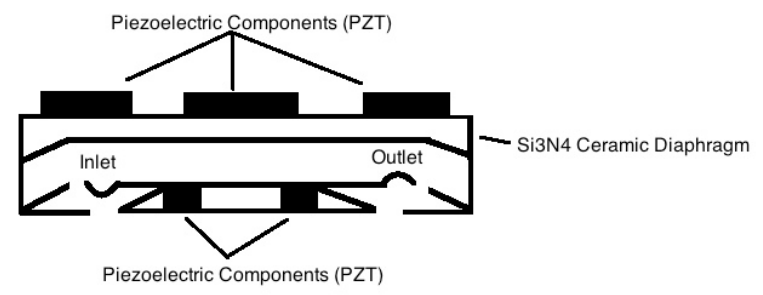

Figure 11: Conceptual design incorporating additional PZT components (Moussa et. al) Dimensions: Membrane

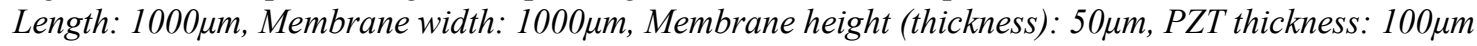




\section{Assumptions}

The main distinctions between ET students design and the Moussa's design are size, location and quantity of the piezoelectric components. Instead of having one large PZT bar at the top, there are three smaller PZT bars throughout the top and an additional two at the bottom between the inlet and outlet valves. With a greater quantity of the piezoelectric component, the expectation is more resonance and, in turn, more bending stress throughout the chip. One thing to be wary of is too much resonance, which could result in inelastic deformation of the $\mathrm{Si}_{3} \mathrm{~N}_{4}$ diaphragm. In their project students assumed the material properties of both $\mathrm{Si}_{3} \mathrm{~N}_{4}$ and PZT5A. Also, the working fluid is water with properties assumed at $70^{\circ} \mathrm{F}$ (density, kinematic and dynamic viscosity). The micro-channel width is $100 \mu \mathrm{m}$. The adjustable flow rate of the pump was set to $5 \mu \mathrm{l} / \mathrm{min}$.

Figures 12 and 13 were generated to give a visual representation of the aim of this project. Figure 12 displays the ceramic and the PZT at rest, and figure 13 displays what happens when an external voltage actuates the piezoelectric. As one can see, the bending stress should be enough to cause turbulence by deflection within the $\mathrm{Si}_{3} \mathrm{~N}_{4}$ membrane. To establish whether or not the flow is turbulent, students also calculated the Reynolds values. The distance between the piezoelectric and the fixed edge $(300 \mu \mathrm{m})$ will be different for this design, considering that there is more than 1 piezoelectric actuator.

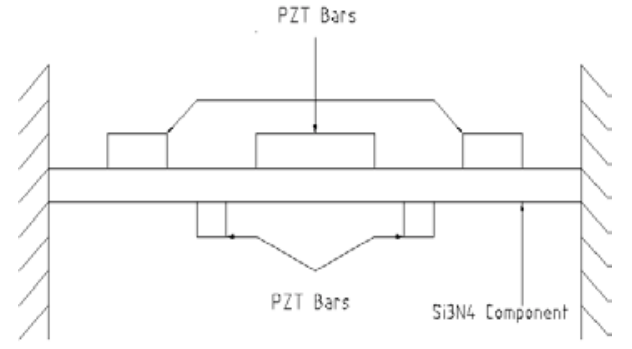

Figure 12: Free body diagram representing piezoelectric bars (non-deformed) attached to the ceramic boundary

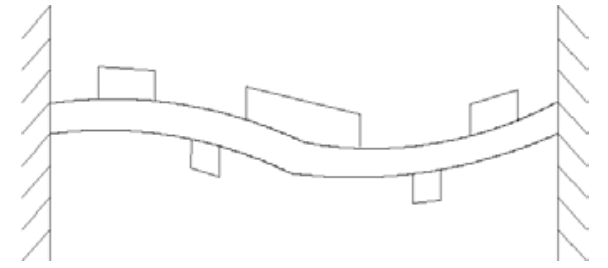

Figure 13: Free body diagram representing the actuated PZT bars deforming the ceramic boundary

\section{Data analysis performed by students:}

As it can be seen from the students' analysis presented below, they used and integrated knowledge from various other courses they previously completed from both mechanical and electrical technology areas. It is to be noted the complexity of notions and concepts they had grasped and the way that they were able to approach it and integrate it in their analysis. Their transition from theory to practical solution and further to conceptual design is also worthy to be emphasized as an accomplishment. Students combined research investigations and studies (literature survey) to create their own concept design, using the newly acquired knowledge to also improve their proposed device. They successfully identified the problems associated with their solutions, proposing new approaches to solve these issues.

The first step in analyzing this problem is determining the flow rate, which is followed by determining the bending stress that is applied to the ceramic by the PZT5A voltage actuator. Because of the general setup of the chip and the various locations of the PZT5A, this is difficult 
to establish. Finally, Reynolds number calculation is used to determine whether or not it is achieved sufficient mixing by means of turbulence within the channel. The formula used for calculating the flow rate, assumes a channel width (D) of $100 \mu \mathrm{m}$ and a pump velocity (v) of $5 \mu \mathrm{L} / \mathrm{min}$. The calculated flow rate for fluid through this micro-channel is $3.93 \mathrm{E}-8 \mu \mathrm{m}^{3} / \mathrm{min}$. The next step was to determine if this design functions as proposed. This can be done by calculating the shear stress of the ceramic component.

Since the design being carried out for this investigation is a conceptual one, students noted several important factors that may or may not influence the outcome of the design. The main issue was to provide a stable bending stress at different locations of the ceramic diaphragm which may be achieved by alternating the voltages for the PZT components located throughout the chip. As a result of this induced bending stress sufficient turbulence is desired to be generated, producing, in turn, mixing within the channel. However, if the voltages are concurrent, or if the voltages are too high, the result will be inelastic deformation of the diaphragm. The primary concern of this design is the fact that turbulence in microfluidics is an area of study that, while researched heavily, has not reached its maximum potential to this point.

Upon completion of the conceptual design, the next step is moving forward with a physical design representing the schematics and material properties outlined above. Once the physical design is established, the necessary testing for the applied voltages and mechanical strength (actual vs. theoretical) can help establish some clearer methods to move forward. The physical design is still under development. Although the students were not able yet to develop a fully functional prototype, the theoretical layout has been established and initial developments are promising.

This above developed section is very important, since it illustrates the complexity of the project undertaken, and the amount of work performed by students during the development of this project. Furthermore it emphasizes the interdisciplinary scope of the project. Moreover, it is a very good tool in assessing the variety of new concepts, theories and notions that the students explored under faculty advisement, above and beyond the topics studied in the "Fluid Power" course in particular, and in courses offered by our Engineering Technology curricula in general. Students demonstrated desire for fundamental research and also found numerous creative ways to apply this research to a practical fluid system.

\section{Conclusions and future developments}

Both teams developed original, innovative and practical solutions in their projects relative to the micro-fluidic devices created. Their solutions were proved to be valuable new experiential activities that can be added to our pool of laboratory activities. This approach stimulated other teams of students to undertake projects that will generate creative experiential activities that may be implemented in our curricula. The heat exchanger experiential set-up was successfully implemented during fall and winter term of AY2012-2013 for Measurement and Instrumentation laboratory (as temperature measurement learning activity) and for "Thermodynamics and Heat Transfer” course (as heat exchanger design and analysis lab activity).

During the development of both student-led projects we monitored the achievement of several learning objectives: (1) students will demonstrate a comprehensive understanding of the concepts, notions and principles taught in the respective course by developing a practical 
solution for and a working prototype of a thermal fluid system of their choice; (2) students will learn how to perform literature surveys pertinent to the project topic chosen, to expand their knowledge beyond the textbook and lectures and to understand the role of research in developing a new innovative system; (3) students will perform market surveys to gain understanding of the existing commercial or industrial systems, their basic characteristics and functionality; (4) to maximize the students' exposure to new and novel technologies, methods and techniques in connection to the topics of the two courses; (5) to stimulate the students' creativity and critical thinking, to reduce their resistance to premature closure and to help students to expand their horizons in areas that are not traditionally linked to engineering or engineering technology.

During this endeavor, students learned about what are the current technologies and the current trends in the micro-fluidics applications field in correlation with thermal-fluid and heat transfer systems, what they need to investigate, what parameters they need to determine, what can be varied, what phenomena needs to be illustrated, how do they should assemble certain systems how to test and validate it, and what can be learned from the project developed.

It is interesting that, once the students were exposed, during laboratory activities to microfluidics theory, methods and fabrication, they developed a real interest in undertaking these two complex projects. Moreover, they "went the extra-mile" to continue these projects beyond the course framework. This exercise proved to be extremely helpful, since same students continued their work in this area and chose their senior design project to be the development of a microfluidic device for pathogen testing.

It should be noted that we successfully achieved our objectives and throughout this paper we presented how well our students proved their in-depth understanding of the topics taught in their respective courses. Both projects stimulated students to create new and novel devices, to seek new concepts that helped them during the project development. They enriched constantly their knowledge and they proved critical thinking and creativity. In addition, this pedagogical approach enabled students to acquire the necessary skills and practices so needed during their capstone senior design projects. Since most of the students are in their pre-junior or junior year, these types of projects, based on open-ended, interdisciplinary projects, offers them a great opportunity to "try-out" at a smaller scale a "capstone type" project, without the pressure that comes with such endeavor.

Students were exposed tremendously to new theories, applications and technologies that most of them are still in the research phase and are not in a textbook or course notes. This type of exposure to what research might entail, did stimulate them further to seek research activities based on their personal or career interests with various faculty members in our department. This approach proved to attract students towards research projects, and to stimulate them to work on innovative projects.

At a more detailed level, students became aware of the problems associated with fabricating an experimental apparatus involving microfluidic devices (miniaturized devices difficult to handle, fluid leaks, bubble formation etc.).

At the end of the term, students involved in the micro-fluidic related projects were asked various small essay type questions. These questions were focused on students input and perception about: (1) the level of understanding of engineering principles being studied during the course 
and how the project was useful in enhancing their knowledge; (2) how well students became aware and gained an appreciation of the phenomena associated with scaling down fluid-thermal processes (challenges and effects such as surface tension and mixing), compared to macro-scale systems; (3) the lessons learned from developing a micro-fluidic device that could serve for different applications, from concept design to prototype, from theoretical calculation to practical tests and validation.

Overall, the feedback received at the end of the term from the participating students was largely positive, students expressed their unanimous appreciation of the superior level of understanding that they gained related to the topics taught in these two courses. As they worked on each project they became more familiar with the issues related to micro-devices and also proposed very interesting and creative solutions to the problems that they approached. Students appreciated the ease with which a prototype can be designed and tested in an attempt to deal with concerns as they arise.

These projects allowed students not only to gain experience with modern techniques, but also to better understand the phenomena associated with various heat transfer and fluid dynamic systems. In addition, students are provided with another opportunity to familiarize themselves with the various micro-scale systems and to grasp the complexity and interdisciplinary character of developing a real-like application or system in particular and of engineering field in general.

\section{References}

1. J.E. Coligan, Commonly Used Detergents Appendix II, Current Protocols in Molecular Biology (Wiley)

2. Y. Feng, Z. Zhou, X. Ye, and J. Xiong, Passive valves based on hydrophobic microfluidics, Sensors and Actuators, A 108 138-143, 2003.

3. M.A. Shoffner, J. Cheng, G.E. Hvichia, L.J. Kricka, and P. Wilding, Chip PCR. I. Surface passivation of microfabricated silicon-glass chips for PCR, Nucleic Acids Research 24, 2 375-379, 1996.

4. N.R. Tax, J. Haneveld, H.V. Jansen, M. Elwenspoek, and A. van den Berg, Capillary filling speed of water in nanochannels, Applied Physics Letters, 85, 15 3274-3276, 2004.

5. P. Griss, H. Andersson, and H. Stemme, Expandable microspheres for handling liquids, Lab Chip 2 117-120, 2002.

6. E. Kjeang, N. Djilali, D.Sinton, Microfluidic fuel cells, Journal of Power Sources 186, 353-369, 2009

7. N. Damean, P.P.L. Regtien, M. Elwenspoek, Heat transfer in a MEMS for microfluidics, Sensors and Actuators, A 105, 137 - 149, 2003

8. W. Lee, W. Fon, B. W. Axelrod, M. L. Roukes, High-sensitivity microfluidic calorimeters for biological and chemical applications, Proceedings of National Academy of Sciences, 106 (42) 18040; doi:10.1073 / pnas.0910433106, 2009

9. L. Wadsö, A. L. Smith, H. Shirazi, S. R. Mulligan, T. Hofelich, The Isothermal Heat Conduction Calorimeter: A Versatile Instrument for Studying Processes in Physics, Chemistry, and Biology, J. of Chemical Education, 78 (8), 2001

10. S. K. Nam, J. K. Kim, S. C. Cho, S. K. Lee, Design and Characterization of a High Resolution Microfluidic Heat Flux Sensor with Thermal Modulation, Sensors, 10, 6594-6611; doi:10.3390/s100706594, 2010 
12. R. S. Figliola, D. E. Beasley, Theory and Design for Mechanical Measurements, $5^{\text {th }}$ ed., Wiley and Sons, 2011

13. Incropera, DeWitt, Bergmand, Lavine, Fundamentals of Heat and Mass Transfer, $7^{\text {th }}$ ed., Wiley and Sons, 2011

14. Y. A. Cengel, M. A. Boles, Thermodynamics an Engineering Approach, McGraw Hill, $7^{\text {th }}$ Edition, 2010

15. J. P. Holman, Experimental Methods for Engineers, $7^{\text {th }}$ ed., McGraw Hill, 2001

16. C. Charyton, J.A. Merrill, Assessing general creativity and creative engineering design in first year engineering students, J. Engineering Education Vol. 98, 2 pp. 145-156, 2009.

17. A. Deshmukh, D. Liepmann, and A. Pisano. "Characterization of a Micro-Mixing, Pumping, and Valving System."

18. G. Hetsroni, A. Mosyak, E. Pogrebnyak, and L. Yarin. "Fluid Flow in Micro-channels." International Journal of Heat and Mass Transfer 48.10 (2005): 1982-998.

19. G. Iaccarino, R. Verzicco. "Immersed Boundary Technique for Turbulent Flow Simulations." Applied Mechanics Reviews 56.3 (2003): 331

20. D. Laser. J., and J. G. Santiago. "A Review of Micropumps." Journal of Micromechanics and Microengineering 14.6 (2004): R35-64

21. W. Moussa, "Simulation of MEMS Piezoelectric Micropump for Biomedical Applications." Autodesk. Center for Mechanical Simulation Technology. Web. 12 Mar. 2012. http://www.algor.com/news_pub/tech_white_papers/mems_micropum

22. J. M. Ottino and Stephen Wiggins. "Introduction: Mixing in Microfluidics." The Royal Society (2004).

23. J. G. Smits, "Piezoelectric Micropump with Three Valves Working Peristaltically," Sensors and Actuators, A2123, pp. 203-206, 1990.

24. D. Tremblay "Resonance in a Piezoelectric Material."Wooster Physics. Physics Department, College of Wooster. Web. 13 Mar. 2012. <http://www3.wooster.edu/physics/jris/Files/Tremblay_Web_article.pdf>. 